\title{
Variable-Focus Fluid Lens Using an Electromagnetic Actuator
}

\author{
J.K. LEE ${ }^{a}$, K.-W. PARK ${ }^{a}$, J.C. CHOI ${ }^{a}$, H.-R. KIM ${ }^{a, b}$ AND S.H. KONG ${ }^{a, b, *}$ \\ ${ }^{a}$ Graduate School of Electrical Engineering and Computer Science, Kyungpook National University, Daegu, Korea \\ ${ }^{b}$ School of Electronics Engineering, Kyungpook National University, Daegu, Korea
}

\begin{abstract}
A variable-focus fluid lens controlled by an electromagnetic actuator and its characteristics are presented in this paper. The repulsive force between an NdFeB magnet and a solenoid through electromagnetic induction causes deflections in the elastomeric membranes integrated in the lens resulting in a focal-length change. The controllability of the focal length of the fabricated lens with an aperture size of $2 \mathrm{~mm}$ was approximately from infinity to $15 \mathrm{~mm}$ (with input current of $100 \mathrm{~mA}$ ). The actuator including the solenoid was carefully designed to avoid thermal crosstalk of the lens fluid and magnet from the electromagnetic induction of the solenoid. The settling time to form a convex shape of the lens membrane was approximately $2.5 \mathrm{~ms}$ at an input current of $20 \mathrm{~mA}$.
\end{abstract}

DOI: 10.12693/APhysPolA.123.202

PACS: $85.85 .+\mathrm{j}$, 42.79.Bh

\section{Introduction}

Conventional focal-length tunable systems relying on mechanically movable elements shown in Fig. 1a are difficult to apply to miniaturized optical devices for consumer applications, such as mobile phones, optical pickup heads and capsule endoscopes, due to the size, power, and cost [1]. Recently, some reliable focus tunable lenses have replaced conventional bulky instruments with small and simple devices such as electrowetting lenses and liquid crystal lenses shown in Fig. 1b [2-4]. However, electrowetting lenses are complex, such as liquid handling, and have time-dependent performance degradation due to surface charge trapping [5]. Liquid crystal lenses consume a large amount of power [6]. Moreover, the responses of these lenses are relatively slow as approximately $100-500 \mathrm{~ms}[6,7]$.

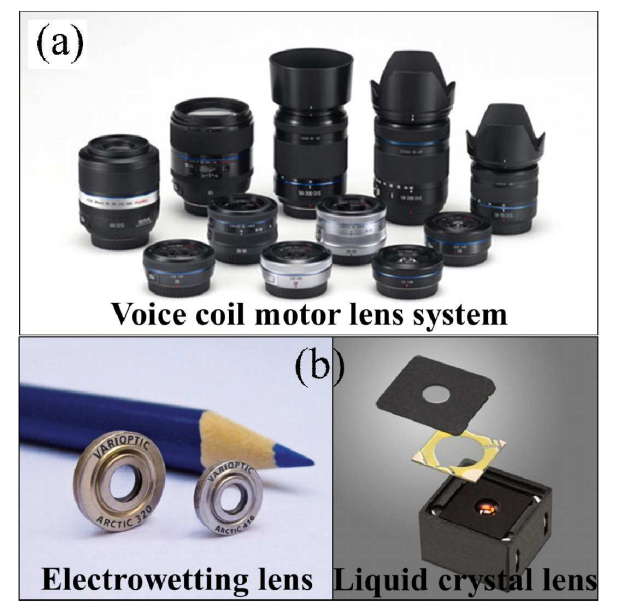

Fig. 1. (a) Conventional focus tunable lens system for bulky applications [11] and (b) miniature focus tunable lenses $[12,13]$.

*corresponding author; e-mail: shkong@knu.ac.kr
Fluid pressure-based liquid lenses are fairly fast as well as simple in terms of fabricating them and handling the liquid [8]. However, although fluid pressure lenses have those advantages, they are difficult to downscale due to the necessity of an external fluid pump to drive the lens. Most fluid pressure lenses need an external pump to achieve a sufficient fluid pressure for a change in the focus [8-10].

A variable-focus liquid lens based on fluid pressure controlled by a fully integrated electromagnetic actuator is presented in this paper. The proposed lens was fabricated using precise polymer machining, micro-assembly, and micro-electro-mechanical-systems (MEMS) technologies. The optical performance of the fabricated lens and characteristics of the actuator are also presented in this paper.

\section{Experimental}

An exploded diagram and photographs of the proposed variable-focus fluid lens with an integrated electromagnetic actuator are shown in Fig. 2. The lens body was fabricated by assembly of four plates: the top plate consisted of a fluidic channel and $50 \mu \mathrm{m}$ thick lens membrane; the first middle plate had a hole for the optical path and a hole with an actuator membrane $(50 \mu \mathrm{m})$ for the magnet actuation; and the second middle plate had a hole for the optical path and a hole for the solenoid housing. The second middle plate was patterned across the solenoid-housing hole to maximize the heat exhaust resulting in low thermal crosstalk between the lens fluid and the magnet. A bottom glass plate enclosed both the lens fluid and actuator. The plates for the lens body were fabricated by the precision three-axis computer numerical control (CNC) machining of polymethyl methacrylate (PMMA) and polycarbonate (PC). Both the lens membrane and the actuator membrane consisted of polydimethylsiloxane (PDMS) (Sylgard 184, Dow Corning, 10:1 ratio of base to curing agent). Irreversible bonding between the PDMS membrane and the polymer body was achieved by $\mathrm{SiO}_{2}$ deposition on PMMA and successive corona treatment with pressure. Assembly between 
the polymer plates was successfully carried out by solvent bonding using cyanoacrylate with corona treatment.

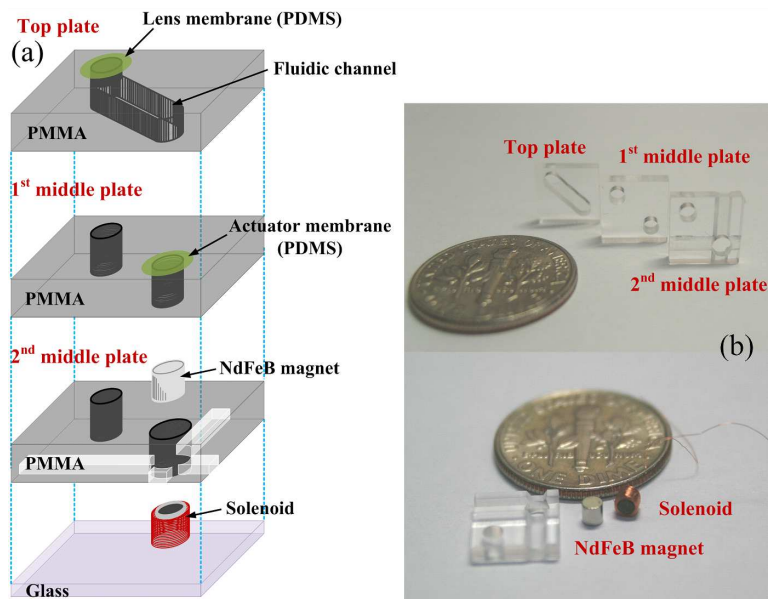

Fig. 2. (a) Exploded diagram of the proposed fluid lens and (b) photographs of the components of the lens.

The electromagnetic actuator consisted of a commercially available $\mathrm{NdFeB}$ magnet and a $\mathrm{Cu}$ coil with a core. $\mathrm{Cu}$ alloy (90 wt\% Cu, $7 \mathrm{wt} \% \mathrm{Ag}$, and $3 \mathrm{wt} \% \mathrm{Sn}$ ) was used for the core due to its low relative permeability value. Since the actuator uses only repulsive force, a ferromagnetic core was unsuitable for the proposed lens. The surface magnetic flux density of the magnet was $0.16 \mathrm{~T}$ and the winding turns $(N)$ of the solenoid was 200 .

Ultrapure water (refractive index, $n=1.33$ ) was used as the lens fluid because of its optical transparency to visible light and low viscosity. Although oil with a high refractive index and thermal stability is attractive as lens fluid, it is difficult to avoid the generation of micro-bubbles and the slow response due to its relatively high viscosity. After assembling the lens body, the lens fluid was encapsulated by the bottom glass plate using ultraviolet-sensitive adhesive bonding (NOA 65, Norland).

Figure 3 shows the fabricated variable-focus fluid lens with black and transparent PMMA plates and its operating principle. The lens size was $8 \mathrm{~mm} \times 8 \mathrm{~mm} \times$ $6.25 \mathrm{~mm}$. The lens fluid was initially dyed to check for leakage of the fluid.

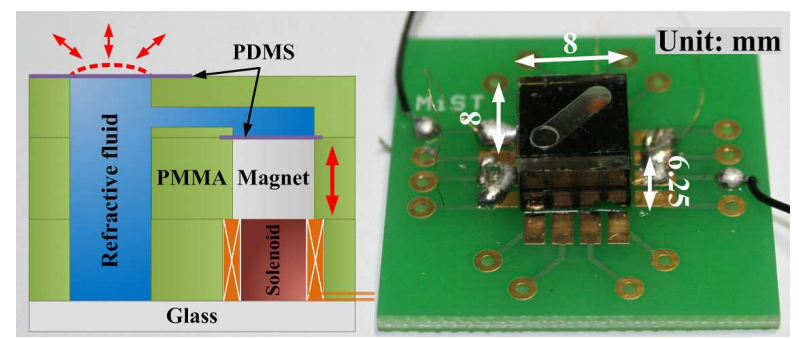

Fig. 3. Fabricated variable-focus fluid lens and operating principle.
The repulsive force and corresponding deformability of the actuator membrane produced by the fabricated electromagnetic actuator were measured using a precision force-strain gauge (RC-1000, Radian). Thermal stability of the actuator was also tested. The performance of the fabricated lens was characterized with an optical transfer function (OTF) image analyzer (Jeewoo Technologies) and high-speed camera (Fastcam MC2 $10 \mathrm{~K}$, Photron).

\section{Results and discussion}

\subsection{Characterization of the fabricated electromagnetic actuator}

The fabricated electromagnetic actuator generates a fluid pressure that corresponds to the deformation of the membrane, produced by the repulsive force between the NdFeB magnet and the solenoid. Therefore, the deformability of the actuator membrane is essential for the controllability of the focal length of the proposed fluid lens. The measured surface magnetic flux density $\left(B_{\mathrm{s}}\right)$ of the magnet was $0.16 \mathrm{~T}$. The amount of magnetic flux density induced by the solenoid determines the repulsive force.

Figure $4 \mathrm{a}$ shows the induced repulsive force as a function of the biased current to the fabricated solenoid. The actuation force was linearly proportional to the current bias as expected. Significantly, a better linearity was observed below $50 \mathrm{~mA}$. Since the lens actuation has to be reversible and predictably controlled, a limited bias current below this current level is available for the proposed lens.

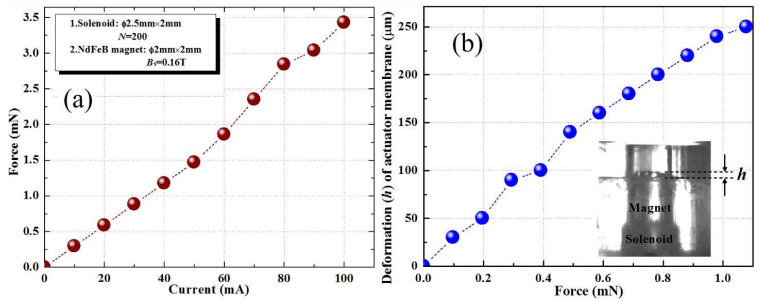

Fig. 4. (a) Repulsive force of the actuator and (b) corresponding deformability of the actuator membrane.

Figure $4 \mathrm{~b}$ shows the deformability of the actuator membrane as a function of the repulsive force produced by the actuator. Although the deformation (height) gradually became saturated due to the restoring force of the membrane as expected, the deformability of the actuator membrane was linear and reversible at a force ranging at $0.5-1.0 \mathrm{mN}$.

The thermal stability of the NdFeB magnet and the solenoid used in this paper were also verified by heat test due to the thermal vulnerability of the magnet. The surface magnetic flux density of the magnet began to decrease above $60^{\circ} \mathrm{C}$. This magnetic degradation rapidly occurred above $80^{\circ} \mathrm{C}$. Fortunately, the solenoid only heated up to $47^{\circ} \mathrm{C}$ until the bias current reached $100 \mathrm{~mA}$. Therefore, the thermal crosstalk of the magnet was insignificant for the fabricated actuator. 


\subsection{Performance of the fabricated fluid lens}

As seen in Fig. 5a, the curvature change of the lens membrane as a function of the bias current was measured. The lens curvature was constantly maintained without obvious fluctuations while the current was applied. The response time of the lens was approximately $2.5 \mathrm{~ms}$. Moreover, variation in the response between a convex shape and a flat shape of the lens membrane was rarely observed in the proposed lens, while a thermopneumatic-actuator-integrated fluid lens has quite notable variations [15].
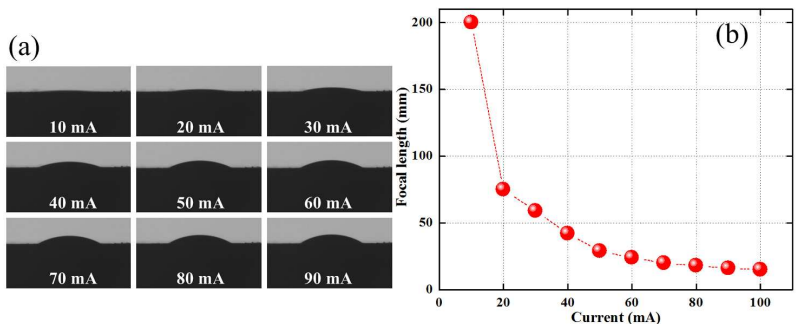

Fig. 5. (a) Curvature change of the lens membrane with a bias current. (b) Controllability of the focal length of the lens.

Figure 5b shows the controllability of the focal length of the fabricated lens. It should be noted that the lens membrane began to deform after the onset of a threshold current. The threshold current of the lens was about $10 \mathrm{~mA}$. The focal length was linearly and appreciably controlled by the actuator until it reached approximately $30 \mathrm{~mm}$. As the current increased above $50 \mathrm{~mA}$, the focal length parabolically shortened resulting in focus saturation. This controllability saturation of the focal length is probably due to reaching a considerable state of restoring forces in the elastomeric membranes. The shortest focal length achieved with our lens was $15 \mathrm{~mm}$.

TABLE

Performance comparison of variable-focus lenses based on fluid pressure.

\begin{tabular}{l|c|c|c|c}
\hline \hline \multicolumn{1}{c|}{$\begin{array}{c}\text { Actuation } \\
\text { type }\end{array}$} & $\begin{array}{c}\text { Aperture } \\
{[\mathrm{mm}]}\end{array}$ & $\begin{array}{c}\text { Focus } \\
\text { tunability } \\
{[\mathrm{mm}]}\end{array}$ & $\begin{array}{c}\text { Response } \\
{[\mathrm{ms}]}\end{array}$ & $\begin{array}{c}\text { Actuator } \\
\text { integra- } \\
\text { tion }\end{array}$ \\
\hline muscular [9] & 20 & $172-41$ & $\mathrm{~N} / \mathrm{R}$ & no \\
piezoelectric [8] & 3 & $\infty-79$ & 2 & no \\
piezoelectric [14] & 2.5 & $500-30$ & $\mathrm{~N} / \mathrm{R}$ & yes \\
thermopneumatic [15] & 2 & $15-3$ & 32,000 & yes \\
electromagnetic [10] & 3 & $\mathrm{~N} / \mathrm{R}$ & $\mathrm{N} / \mathrm{R}$ & no \\
electromagnetic & 2 & $\infty-15$ & 2.5 & yes \\
[this work] & & & &
\end{tabular}

Table compares the performance of variable-focus lenses based on fluid pressure. The proposed lens with the integrated electromagnetic actuator had better performance than earlier reported actuator-integrated fluid lenses.

\section{Conclusions}

In this paper, a variable focus fluid lens based on fluid pressure induced by an electromagnetic actuator is presented. The actuator consisting of an NdFeB magnet, a solenoid, and a PDMS membrane was integrated into the fluidic channel. The repulsive force of the actuator induced a curvature change of the lens membrane placed on the fluidic channel filled with a refractive fluid. Maximum focal-length tunability of the fabricated lens with an aperture size of $2 \mathrm{~mm}$ was from infinity $\mathrm{mm}$ to about $15 \mathrm{~mm}$. The response time to form a convex lens was about $2.5 \mathrm{~ms}$ with an input current of $20 \mathrm{~mA}$. The fabricated lens rarely had any thermal crosstalk during its operation.

\section{Acknowledgments}

This work was supported by the National Research Foundation of Korea Grant funded by the Korean Government (2012-0000629 and the Human Resource Training Project for Regional Innovation).

\section{References}

[1] C. Cavallotti, M. Piccigallo, E. Susilo, P. Valdastri, A. Menciassi, Paolo Dario, Sens. Actuators A 156, 72 (2009)

[2] B. Hendriks, S. Kuiper, IEEE Spectrum 41, 32 (2004).

[3] J.K. Lee, K.-W. Park, H.-R. Kim, S.H. Kong, Jpn. J. Appl. Phys. 49, 06GN11 (2010)

[4] K. Asatryan, T. Galstian, A. Bagramyan, V. Presniakov, A. Tork, A. Zohrabyan, P.P. Clark, U.S. Patent Appl. No. 12/996,593, 21 April 2011.

[5] M. Maillard, J. Legrand, B. Berge, Langmuir 25, $6162(2009)$

[6] ww.tessera.com.

[7] B. Berge, Nikkei Electron. 911, 129 (2005).

[8] H. Oku, M. Ishikawa, Appl. Phys. Lett. 94, 221108 (2009)

[9] D.Y. Zhang, V. Lien, Y. Berdichevsky, J. Choi, Y.H. Lo, Appl. Phys. Lett. 82, 3171 (2003)

[10] S.W. Lee, S.S. Lee, Appl. Phys. Lett. 90, 121129 (2007)

[11] www.samsung.com .

[12] ww.varioptic.com.

[13] Www.lensvector.com.

[14] F. Schneider, J. Draheim, C. Muller, U. Wallrabe, Sens. Actuators A 154, 316 (2009).

[15] W. Zhang, K. Aljasem, H. Zappe, A. Seifert, Opt. Expr. 19, 2347 (2011). 